\title{
Filigrane
}

Écoutes psychanalytiques

\section{À propos de Lettres du divan}

\section{Louise Grenier}

Volume 26, numéro 2, 2017

URI : https://id.erudit.org/iderudit/1055363ar

DOI : https://doi.org/10.7202/1055363ar

Aller au sommaire du numéro

\section{Éditeur(s)}

Revue Santé mentale au Québec

ISSN

1192-1412 (imprimé)

1911-4656 (numérique)

Découvrir la revue

Citer ce compte rendu

Grenier, L. (2017). Compte rendu de [À propos de Lettres du divan]. Filigrane, 26(2), 105-115. https://doi.org/10.7202/1055363ar d'utilisation que vous pouvez consulter en ligne.

https://apropos.erudit.org/fr/usagers/politique-dutilisation/ 


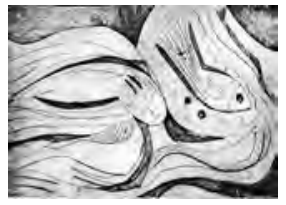

\section{À propos de Lettres du divan}

\section{Louise Grenier}

Soir des mots - sourciers dans le silence. (Celan, p. 75)

a seule façon de lui dire que je l'aimais avait été de lui écrire. Mais il fallait étoffer la lettre, lui donner corps, l'incarner dans une langue ignorée, maternelle en fait. Non pas me lamenter admirablement à la manière de $L a$ religieuse portugaise ou d'Héloïse, non pas poétiser un amour à la dérive, mais dire ce qui ne cesse de me fuir, de disparaître. Parlant, je l'éloignais de moi, écrivant, je me rapprochais de lui. Comme si les mots avaient ce pouvoir de l'effacer, de le ramener. Les mots dits ne trouvaient en lui que le lointain, l'étranger. Les mots écrits creusaient l'intime, le familier. Parlant, je perdais ma trace, et mes mots se disloquaient derrière moi. Écrivant, je retrouvais ma trace, et mes mots se recomposaient sur ses bords. Dans le passage de la parole à l'écrit, une lettre s'échappait du divan, errante en quelque sorte.

Dans ce livre, Lettres $d u$ divan, analystes et analysants s'interrogent sur la place à accorder aux écrits en cours d'analyse ou après celle-ci. Lettres ou courriels adressés à l'analyste sont une halte sur «les sentiers du silence ${ }^{1}$ ». Pour aller vers ce qui recommence dans le transfert, pour aller vers un dire élémentaire qui serait dissimulé sous un amoncellement de signes. Sans pallier les lacunes de la parole, la lettre, indépendamment de son contenu, fait signe, et dans ses enchaînements et répétitions, pourrait bien être une façon d'échapper à l'absence, la sienne, celle de l'autre.

$\mathrm{Au}$ commencement, Freud s'était fait le romancier de ses belles hystériques: Anna, Dora, Élisabeth, Lucy et les autres. Le littéraire ouvrant ce nouveau champ d'un savoir en gestation, la psychanalyse. La psychanalyse n'est-elle pas fille de l'écriture comme me l'a si joliment rappelé Isabelle Lasvergnas? Car Freud n'écrit pas seulement de beaux "contes cliniques» mais des lettres, et dans ces lettres passionnantes, notamment à Fliess, il y a un saut de, et pour la pensée qui s'opère aussi pour nous. Cette correspondance unique en son genre reflète bien le lien affectif qui l'unit à son ami. Comme si la passion d'amour ou d'amitié tenait au lointain, comme si les 
mots tiraient leur pouvoir de faire revenir un disparu, et dans le cas de Freud, de pointer en direction d'un lecteur du futur. Écrivant à Fliess, Freud s'adressait déjà à nous, initiant par-là un transfert qui nous fera entrer en analyse.

Après mes rencontres de Cerisy, puis avec Jean-François Chiantaretto et son groupe Littérature personnelle et psychanalyse, j'ai eu envie de parler de ces lettres qui nous parviennent du divan ou d'ailleurs, poèmes ou récits d'enfance, journaux intimes, comme "des cartes postales» (Mireille Fognini) d'un pays étranger. La lettre, même virtuelle, a elle aussi cette dimension d'étrangeté. Elle émane d'un no man's land du sujet, peut-être déjà traversé d'ailleurs, et qui lui donne le sentiment de s'être oublié quelque part. Où? Dans une zone psychique sans nom et sans frontière, non cartographiée. Pour une de mes patientes très défensive, soucieuse de conserver une illusion d'omnipotence, la poésie lue en séance ou envoyée par message électronique fut un moyen de préserver cette faille intime pour en faire la matière d'une œuvre.

\section{La lettre}

Ghislaine Pesant, poète et analysante, me semble avoir exprimé d'une façon saisissante le motif sous-jacent des Lettres du divan.

Son poème «Fragments d'autopsygraphie», commence ainsi:

Quand on parle

une petite chose s'en écrit

et tu tentes de l'écrire

Écrire pour retrouver les traces

de ce qui s'est parlé ou non

Ou s'est entendu

peut-on retourner à la lettre perdue

d'amour de l'amour...

Quelle est cette "petite chose» inscrite comme une trace signifiante d'une rencontre avec l'autre parlant? Ça pourrait être un mot, une lettre, une ponctuation, quelque chose d'ineffaçable et d'essentiel pour retrouver l'ossature d'une mémoire inconsciente de soi. Un signe en direction d'une lettre perdue, de ce que Je suis pour l'autre. À défaut de cet écrit mythique, Je n'existe pas. Pareil en séance de psychothérapie ou de psychanalyse où la 
parole a des effets inattendus tant chez l'analyste que l'analysant. De l'autre côté de soi, c'est l'écoute de l'analyste qui attire l'infantile au-dehors, c'est cette écoute si particulière qui permet l'émergence de mots oubliés, d'émotions rejetées, d'un certain état de l'être en mal de reconnaissance. En ce sens, l'association libre est déjà un début d'inscription signifiante, ou ne serait-ce pas plutôt une réinscription? Le divan est ici le symbole d'un lieu où la parole recouvre les silences d'anciens tracés. Cette petite chose qui s'est inscrite, peut-être à ton insu, pendant que tu parlais à ton analyste, s'inscrit par-dessus. C'est cela aussi une lettre du divan.

C'est cela, mais pas seulement, écoutons encore une fois Ghislaine Pesant:

le cœur endeuillé de l'enfant de neuf ans dans une mère couchée en moi sur le divan-lit

C'est par cette image troublante d'un cœur endeuillé pour deux, mère et fille, l'une dans l'autre, dans un corps étendu sur le divan, que la poète se souvient de son analyse et nous donne à lire ce qu'elle dit là. Je n'ai pu résister au plaisir de commenter ce passage car il me semble symboliser un fondement possible de ce livre, à savoir l'unité première, archaïque, du dit et de l'écrit, ou d'une lettre prise dans la matière même de la scène incestueuse.

Il y aurait donc des "mots écrits à l'encre invisible mais je n'ai pas encore trouvé le moyen de les faire apparaître», écrit Louise Gareau dans un moment d'angoisse. Elle appelle à l'aide son analyste qui, faute de disponibilité immédiate, lui propose d'écrire. Voilà pour elle où commence l'écriture qui, trois jours plus tard, deviendra poésie de la main gauche. Geste de coupure du lien imaginaire - matriciel? -, geste séparateur aussi, qui permet la symbolisation de ce qu'elle appelle «l'indicible». La métaphore exprime bien qu'il existe un déjà écrit inconscient, hors de la vue de son auteur, et qui poursuit «la parole adressée» à son analyste:

J'écris comme le vent

Rallume les étoiles

J'écris comme le fleuve

Épanche les rivières [...] (p. 74)

Il est en de même pour Rose qui, poète, trouve en Alejandra Pizarnik ${ }^{2}$ une âme sœur pour soutenir son désir d'écriture. Rose m'adresse des lettres 
qui sont dans le prolongement de ses séances. Elle dit là ce qui a été écrit «à l'encre invisible» sur le divan. Son transfert, et tout ce qu'il charrie de ses tourments amoureux, peut ainsi être vécu par elle sans qu'il n'y paraisse. À l'analyste de savoir lire à défaut d'entendre, semble-t-elle me dire. À l'analyste aussi de savoir déplacer son écoute du dit à l'écrit pour traduire in vivo ce qui se trame entre les lignes de la parole. Alejandra Pizarnik envoyait depuis Paris des poèmes, des lettres à son ex-analyste, León Ostrov, ce qui était une façon de le poétiser et sans doute de remédier à l'absence.

Dans la présentation de Lettres du divan, j'ai posé cette question: «L'écrit du divan - poèmes, lettres ou récits de rêves - serait-il une façon de s'éloigner de ses propres traces, de l'infantile, ou au contraire de s'en rapprocher? Écrire pour se sentir vivant, existant, ou pour se réfugier dans les mots comme dans la mère?» Mais attention, méfions-nous des formules. Dans l'écriture comme dans la parole, nous finissons toujours par nous heurter à un mur. Une absence, dirais-je. L'absence est un mur invisible, infranchissable forcément. Écoutons Jean-François Chiantaretto parlant de l'existence-limite de certains patients: «Et vivre revient ainsi à écrire indéfiniment un destin déjà écrit.» Se faisant poète de leur douleur d'être, ou de ne pas être, il montre bien que lorsque «les bras levés du désir «(Rusbroek) ${ }^{3}$ frappent le vide, c'est tout l'être qui tombe. Il n'y a pas de limite parce qu'il n'y a personne de secourable devant soi, en soi. Rien qui vous tient, ou à qui tenir. Restent les mots pour tenir encore un peu.

Comment soigner la folie

De l'autre en soi

Sans perdre l'autre en soi

Et son envie de la vie?

L'analysant-poète est plus ou moins toujours sur son départ, au plus près d'une fin jamais annoncée, pas toujours survécue. Francine Godin se voyait en analyse jusqu'à la fin de sa vie. Sa vie à elle. Lui ne mourrait pas. C'est dans l'image des bêtes chassées par leur mère qu'elle trouve la force de s'arracher à celui qu'elle considère comme sa mère, son père, tout en Un. Est-il vraiment possible de se quitter soi-même, peut-on lire entre les lignes? Les mots se mêlent, la phrase se détache, le discours se découd, il a perdu son centre.

Chez Mireille Fognini, la poésie se glisse entre théorie et clinique. Elle se demande où sont passées ces cartes postales qui constituent un signe 
d'existence adressé à son analyste: je pense à vous, disent les lettres, et vous? N'est-il pas important qu'il (ou elle) accuse réception des envois de ses patients? Oui, répond-elle. Comme pour les rêves ou les dessins d'enfants, c'est une matière vivante qui s'introduit là, entre les mots parlés, un écrit intime issu de ce qu'elle appelle «l'aire encore ensauvagée d'une non-parole, une terra incognita où la représentation partageable $[. .$.$] demeure encore$ en friche dangereuse emplie d'inconnu, frappée peut-être même d'interdits, de tabous, de non-symbolisés ou de symbolisables, voire d'encryptements somatopsychiques, de forclusions [...]»(p.46-47). Comment faire de la parole avec un tel objet, se demande-t-elle? et quelles sont les conséquences d'une non-prise en compte de ces productions «laissées en dormance, voire enterrées en poche restante de l'analyste [...]» (p. 49). Dans la vignette clinique qui illustre sa réflexion, Mireille Fognini émet l'hypothèse que l'écriture pourrait avoir la fonction cryptophore d'un trauma originaire ou transgénérationnel. Ces écrits du divan seraient-ils des copies, ou des tentatives de transcription d'un «fait réalitaire» (Maria Torok), frappé d'interdit de communication. Autrement dit d'un passé qui serait «présent dans le sujet comme un bloc de réalité» (Abraham et Torok, 1987, p. 255)?

Qu'est-ce qui dans un énoncé singulier, une image incongrue dans la cure, permet l'instauration subjective? Est-ce le trait unaire, ce trait connoté du Réel, en tant qu'il renvoie l'être parlant à ce qui lui échappe dans son dire? Telle est la question posée par Monique Lévesque qui rapporte cet énoncé lancé par une analysante: «Il y a une mare de sang sur votre tapis.» Voilà qui ressemble en effet à un ovni dans le cours de la parole. Au commencement de l'écriture, il y a l'encoche, le marquage des repères et des objets, la matérialité du signe, rappelle-t-elle. Et s'il est vrai que l'écriture précède la lecture, prendre la parole consisterait-il à déchiffrer, traduire nos inscriptions mémorielles? Le trait initial, séparateur, demeurant quand même inaccessible au verbe. En ce sens, l'énoncé qui jaillit de la bouche de son analysante est de la matière signifiante brute. Voilà qui pour moi évoque aussi le poète qui se laisse traverser, voire investir, par les mots, et qui n'a pas besoin de savoir ce que ça veut dire pour que ça existe.

Marie Hazan n'aura peut-être envoyé qu'une seule lettre à son analyste, celle qui constitue un des chapitres de ce livre. Elle boucle la boucle d'un parcours qui passe par son enfance à Beyrouth où règne la violence sociale et, plus prégnante pour elle, plus blessante, celle des petites filles qui se moquent de sa différence. La terreur enkystée psychiquement n'est pas celle de la guerre et des conflits sociaux du Liban, mais la férocité inattendue 
de ses pairs. Son texte dessine la carte de ses multiples déplacements et rencontres où il s'agit de revenir vers son analyste, la Dame blonde, comme vers l'enfant qu'elle aura été, une petite fille blonde, une proie trop facile, dit-elle.

"Nous étions deux absents en présence l'un sans l'autre», note Claude Spielmann à propos d'une jeune patiente qu'il appelle Ophélie. Trop de transfert, ça étouffe et ça ne se dit pas. Est-ce que ça s'écrit? Dans ce cas, c'est l'analyste qui en ressent le besoin, question d'instaurer du tiers, de faire un appel à témoin. Ophélie, ô ma folie, dérive dans le regard noyé de son analyste. Elle est celle qui éternise la peine d'amour. Ophélie flotte dans nos regards noyés de chagrin. Et lui, devant elle, est en deuil de cette femme-là qui symbolise ses propres disparus. Car elle appartient à une lignée d'absents, hommes ou femmes, qui ont constitué son existence. À ses fantômes qui actualisent la nécessité de l'écriture, il donnera le beau nom de Sarah.

Au revers de l'écrit, un enfant est mort. Marie-Brigitte Ruel croise dans l'imaginaire une dame qui ressemble à Lady Chatterly. Une femme amoureuse "sur l'allée cavalière», comme "une petite sœur perdue que la vie aurait éloignée» à jamais. C'est qu'il faut porter sa croix, faire l'ange, se sacrifier pour qui ? La lecture de D.-H. Laurence ouvre son imaginaire alors que l'analyse lui permet de passer du nous fusionnel au Je muet, puis au Je parlant. C'est l'analyse qui pousse à l'écriture, ne serait-ce que pour canaliser un transfert passionnel ou paternel. Des pages manuscrites rythment sa vie entre, et pendant les séances, comme des alliées indispensables pour surmonter ses ambivalences et ses résistances, dit-elle. L'amour a partie liée avec l'écriture, toujours, comme avec la mort.

Claude Brodeur se dédouble, se fait son propre analyste, revient sur les lieux d'un trauma, la mort encore, celle d'une petite sœur encore, la mort et l'absolue nécessité de vivre quand même, de vivre surtout, dans et par les mots qui nous humanisent. C'est dans la fiction qu'il rejoint tous les temps du désir, y compris dans cette scène où le père si proche de la petite sœur mourante lui indique la voie de son propre désir. Un désir de se retrouver que l'écriture réalise en quelque sorte.

La mort aurait-elle partie liée avec l'écriture? Pour Daniel Puskas, il y avait d'abord les écrits de son analyste et de son superviseur. Ils écrivent, dit-il, et moi, du plus loin que je me souvienne, «je ne voulais pas mourir» (p. 187). L'écriture en diffère le moment, elle est un appel à l'autre, une sorte d'atelier où un auteur dessine des formes et des visages. Il s'agit de créer, d'inventer, et pourquoi pas, de raconter une cure sous forme de fiction. Lui aussi cherche la lettre, celle qui rend toutes les autres possibles, 
le fin mot d'un dire qui saigne, qui heurte. Qui met au monde aussi. La vie psychique est peuplée de lettres, d'inscriptions; certaines très anciennes, invisibles, muettes, comme celles des grottes de Lascaux qui dessinent notre préhistoire, ou des Inuksuit (pour les Inuits) sculptés dans la pierre, repères géographiques qui symbolisent bien la pérennité du lien d'entraide ou sa nécessité.

L'enfant est seul en face des mots qui sont pour lui des entités abstraites, étrangères. Des "monuments», comme le rappelle également Isabelle Lasvergnas. Sur les mots, on ne peut que buter ou se casser la gueule. C'est dans l'expérience du passage à l'écriture, dans la parole aussi, qu'elle-même se heurte à un obstacle angoissant. Les mots sont toujours en avance sur l'enfant. L'enfant, c'est bien connu, est porté par le langage autant que par les bras d'une mère. Ça parle et ça s'écrit en lui, ça laisse des marques qu'il prendra une vie à traduire. Il y a des moments privilégiés où il arrive à rejoindre les mots de l'autre, des moments qui «s'approchent de la temporalité psychique primaire», rappelle Isabelle Lasvergnas (p. 135). Dans et par l'écriture, elle rêve de rejoindre minimalement «le mouvement de conquête inaugurale» de la naissance de l'enfant à la parole. Pour elle, il s'agit de s'abstraire au sens d'une coupure d'avec le corps matriciel refoulé et de «[t]irer de la mère l'infantile du mot» (p. 139). Peut-être y a-t-il chez l'analysant qui écrit, ce rêve de traverser les silences de la mère ou d'entendre une voix qui aurait été la sienne. J'ajouterais que parler, c'est aussi se tirer de la mère. Il y aurait dans les mots un noyau de silence, et ce silence inaltérable, l'analyste l'incarne mais n'en sait rien.

Chez Janine Altounian, l'écriture ne cesse de vouloir dire la souffrance des siens, de la mère notamment, cette mère qui ne connaissait pas la langue de la tendresse, seulement celle de la survie. Après chaque séance, elle transcrit les propos qu'elles ont tenus, elle et son analyste. L'écrit apparaît ici comme un mémorial, une façon de retenir l'éphémère d'une rencontre; l'analyste étant à la fois le destinataire supposé et la cause de cette mise en récit. Une seconde forme d'écriture, celle «destinée à la publication, se situe au croisement de l'autobiographie, de la réflexion analytique et de la transmission des traumas collectifs de l'histoire» (Altounian, p. 14). Il est important pour Janine Altounian de socialiser une subjectivité qui émerge dans la cure, de la faire exister avec d'autres - les parents muets - et pour d'autres. Étrange le fait qu'elle doive préciser qu'elle n'est pas analyste. Il est vrai que son écriture ne cesse d'être analysante, de la faire revenir dans les zones sinistrées de son histoire et d'en faire la théorie. 
L'écriture se présente comme une nécessité existentielle pour certains de nos patients, une façon de s'établir dans l'être, et peut-être aussi de s'assurer de notre existence. Une sorte de jeu de for-da: «je te fais disparaitre, je te ramène à moi, et pour cela, il faut que je t'intéresse avec mes récits ou mes rêves». Ce sont les mille et une séances recommencées, réécrites, prolongées. La fin est ainsi différée. Il y a toujours une suite à mon histoire, et si le passé inspire particulièrement nos écrivants, c'est peut-être parce que l'avenir est impensable. "Pas encore, encore un peu...», murmure la lettre.

Il me semble que la patiente d'Evelyne Gosse-Oudard, celle qui revient pour lui demander d'écrire avec elle un livre, veut elle aussi contourner la séparation, mais pas seulement. Imaginons l'analyste en co-auteure d'un récit traumatique, ce qu'elle est sans doute déjà dans l'imaginaire de cette femme. Celle-ci n'est sortie du silence noir des placards et de l'absence maternelle que pour entrer dans la terreur des mots qui lui étaient étrangers. Assise devant son analyste, elle lit à haute voix des chapitres de son histoire semée d'abandons et de violences. Un jour, ce sera trop dur, elle s'interrompt. L'analyste poursuivra devant elle la lecture de son texte, lui prêtant sa voix. Une lecture à deux voix, l'une faisant résonner l'émotion de la première, actualisant un moment traversé sans être vécu, réalisé, en somme. Lisant à haute voix le texte de sa patiente, l'analyste occupe la place du proche secourable, dans l'ombre de la mère absente, ajouterais-je. Un tiers surgit là, dans ce creux, dans cette faille insoutenable de l'être (Kundera), trouvant-créant du tiers et annonçant, sinon une séparation, du moins la possibilité d'y penser.

Dans le martellement produit par le slam des adolescents, les mots claquent entre les silences, comme des portes battantes, ou comme un jeu qui concrétise au quotidien l'amertume du monde. Sophie Lapointe donne l'exemple d'un pré-adolescent qui dessinait en séance les contours d'un visage sans rien dedans. Cette forme vide apparaissait parmi d'autres personnages, c'était son visage absent, bien sûr. Dans la clinique adolescente, les textos ou SMS, et les réseaux sociaux prédominent en tant que modes de communication, ce qui se reflète dans la communication avec celle qu'ils nomment une psy. Ils n'écrivent pas de lettres au sens strict mais des informations, des mouvements d'humeur, des émoticons, des signes. L'autre virtuel est toujours à sa disposition, une illusion bien sûr, car ce qui est raté ici, c'est encore la castration symbolique et la reconnaissance de l'altérité. Comme si dans l'imaginaire, les places de l'un et de l'autre se chevauchaient et que les mots étaient lancés comme des choses à la face de l'autre. 
Voilà peut-être ce que laisse aussi entendre Michel Peterson quand il se dit submergé par l'afflux de mots d'une patiente aux prises avec un transfert érotique massif difficile à analyser. Lui-même avoue avoir apporté à une de ses analystes quelques deux cents pages qu'elle lui remit un mois plus tard en lui disant qu'elle n'y comprenait rien. Trop subtil, trop abondant! Devenu analyste, lui-même n'a jamais repoussé les textes qui lui étaient adressés. Y répondre n'est pas sans risque, dit-il. Certains passages pourront être utilisés contre vous en cas de conflit avec l'institution ou avec un patient. Danger également d'être violenté, «monstrualisé» par les mots de l'autre au sens où ils servent non la symbolisation ou l'élaboration psychique, mais la mise en acte pulsionnelle. La haine trouve ainsi une issue passionnelle. Comment l'élever à la dimension du fantasme pour la rendre "parlable», partageable? Comment opérer une séparation entre les espaces du dehors et du dedans, de l'intime et de «l'extime», de soi et de l'autre? L'écriture de l'analyste, qu'elle soit clinique, théorique ou littéraire, poursuit le travail de la cure. Dans la foulée de Lacan et Derrida, l'écriture analysante «est toujours déjà une archi-écriture et, à ce titre elle rature la présence sans jamais oublier la parole vive de la séance [...]» souligne Michel Peterson (p. 164-165). Sans oublier qu'en son principe cette archi-écriture ${ }^{4}$ nous introduit à la mort. La mort qui n'est pas de l'ordre du pensable mais qui donne à penser.

La mort de son analyste, à défaut d'en parler, il faut bien en écrire. Sans doute agit-elle en catimini dans le lien transférentiel comme dans tout lien d'importance, toujours intolérable. Luiz Eduardo Prado de Oliveira appuie ses réflexions sur l'article de Winnicott concernant la haine dans le contretransfert et sur l'étrange relation qu'ont longtemps entretenue Winnicott et Masud Khan, analysant devenu disciple et ami. Ailleurs, dans l'histoire de la psychanalyse, la haine, autre visage de la mort, s'est insinuée entre Mélanie Klein et sa fille Melitta, de même que dans le fonctionnement des écoles et groupes analytiques. L'inanalysé d'un individu ou d'un groupe se manifeste par des agirs de rejet ou de rupture. Il arrive aussi qu'un écrit, adressé à son analyste ou publié, permette l'élaboration psychique de la haine, et tout aussi bien de la haine de soi. Eduardo Prado de Oliveira nous rappelle que selon Freud, la haine «est plus ancienne que l'amour». Et si son rôle est premier, fondateur du lien, c'est à condition qu'elle puisse nous faire croire en l'amour, conclut-il.

Tout au long de la rédaction de cet ouvrage, les mêmes questions furent soulevées. Quelle place accorder aux écrits du divan dans le travail analytique? Faut-il les considérer comme un matériel à interpréter au même titre 
que le rêve ou le symptôme, ou les reléguer hors de l'analyse et les traiter uniquement comme une production du Moi? Le fait d'écrire à son analyste, ou pour lui ou elle, est-ce une façon de se maintenir dans un non-dit venu du fond des âges, ou au contraire une façon de le rejoindre?

Jacqueline Rousseau-Dujardin y répond sans ambiguité. Elle lit les écrits qui lui sont adressés mais elle n'en parle pas car, dit-elle, ils ne font pas partie du matériel des séances. Cette position, elle l'appuie sur la méthode fondée par le Freud des Études sur l'hystérie, des Cinq psychanalyses et de L'interprétation $d u$ rêve. La psychanalyse étant dans ses fondements et par sa méthode une "cure par la parole», l'écrit n'y a pas sa place, ajoute-t-elle. Et en effet, le respect de la règle fondamentale des associations libres est ce qui permet l'émergence de l'inattendu dans une cure, le retour du refoulé, et un transfert qui suppose la neutralité de l'analyste, son identification à sa fonction d'interprète. Soulignant à juste titre l'importance des «virages de la pensée», des lapsus ou actes manqués, des rêves et autres trébuchements du discours, pour qu'émerge les souvenirs, elle marque l'écart entre la parole et l'écrit pour souligner la spécificité de la première.

Certes, il ne faut pas confondre la parole analytique avec ce que j'ai d'abord appelé «les écrits du divan». Sur ce point, les auteurs de ce recueil seront d'accord: toute analyse se fonde d'abord sur la parole et sur l'écoute. En marge de la parole, l'écrit, adressé ou non à l'analyste, est ce trait qui à la fois institue l'altérité en la personne du lecteur et permet de différer la mort. L'écrit ouvre un espace transitionnel et à ce titre, mérite d'être inséré dans le tissu de la parole à l'instar du dessin ou du rêve. La scène de l'écriture pourrait aussi dériver de la désespérance à faire entendre la demande inconsciente qui sous-tend toute parole; une demande de reconnaissance, mais par qui? Dans cet article, j'ai voulu tirer un fil qui, en traversant tous les textes de Lettres du divan, attraperait les subjectivités qu'il rencontre sur son passage. Un fil glissé dans le texte comme dans un tissu, pour y dessiner un visage, une pensée, une lettre, un je ne sais quoi.

Louise Grenier, Montréal louisegrenier.c@gmail.com

\section{Notes}

1. M. Heidegger: «Qui d'entre nous, [...] pourrait s'imaginer que sa tentative de penser est à demeure sur le sentier du silence", Lettre sur l'humanisme. GA 9, 344, p. 113.

2. Alejandra Pizarnik (1936-1972) est une poète argentine célèbre dont on a publié la correspondance avec son analyste. 
3. «Et les bras levés du désir n'atteignent jamais la plénitude adorée.»

4. Pour définir son concept d'archi-écriture, Derrida doit le distinguer de l'écriture usuelle (phonétique, alphabétique, vulgaire). Cette dernière s'impose par dissimulation de l'archi-écriture. L'archi-écriture ouvre le rapport à l'autre comme condition de tout système linguistique, tandis que l'écriture chasse son autre.

\section{Références}

Abraham, N. et Torok, M. (1987). L'écorce et le noyau. Paris: Flammarion.

Celan, P. (1991). Soir des mots. Dans De seuil en seuil (trad. V. Briet). Paris: Christian Bourgois.

Grenier, L. (dir.) (2017). Lettres du divan. Écrire à son psychanalyste. Montréal: Liber.

Heidegger, M. (1946). Lettre sur l'humanisme.

Pizarnik, A. (2016). Correspondance avec León Ostrov, 1955-1966. Paris: des Busclats. 